\title{
Sepsis Antibacterial Therapy Perspective Possibilities of Sepsis Curing, Using Drugs that Activate the Cholinergic Anti-Inflammatory Pathway
}

\section{Pavel Franzevich Zabrodskii*}

Saratov Medical University "REAVIZ", Saratov, Russia

*Corresponding Author: Pavel Franzevich Zabrodskii, Saratov Medical University "REAVIZ", Saratov, Russia.

Received: October 14, 2019; Published: October 28, 2019

DOI: $10.31080 /$ ASMI.2019.02.0413

\begin{abstract}
Literature data, as well as the results of our experimental studies (after the cholinergic anti-inflammatory mechanism - «cholinergic anti-inflammatory pathway» - we established in 1987) allow us to suppose, that n-cholinomimetic ( $\alpha 7 n A C h R s$ agonists, nicotine), acetylcholinesterase reversible inhibitor, adrenomimetics ( $\beta 2$ ARs agonists) and m-cholinomimetic (m1-cholinomimetics), along with a range of therapeutic measures, in particular, with prescription of antibiotics, can be considered as promising agents for the treatment of sepsis and septic shock.

Keywords: Cholinergic Anti-Inflammatory Pathway; Sepsis; $\alpha 7$ nachr Agonist; $\beta 2$ ars Agonist; Acetylcholinesterase Reversible Inhibitor; Proinflammatory Cytokines
\end{abstract}

\section{Sepsis antibacterial therapy}

Septicaemia and septic shock are a major public health problem. Every year, around the world, it causes the death of more than a million people. Mortality from sepsis, depending on various factors, ranges from 12 to $60 \%$ of all deaths associated with diseases and their complications [1], and there is an increase in the number of cases of sepsis and the mortality rate from it [2]. Sepsis is a clinical syndrome with the development of life-threatening organ dysfunction caused by impaired regulation of response to infection. In septic shock, there is a critical reduction in tissue perfusion; many organs, including lungs, kidneys and liver, can also be severely affected. The most common causes in immunocompetent patients are various types of gram-positive and gram-negative bacteria. Atypical bacterial or fungal infections may be contributing factors in immunodeficient patients. Symptoms include fever, hypotension, oliguria and blurred consciousness. Diagnosis is based on clinical studies combined with bacteriological sowing results indicating the presence of infection; early detection and treatment are crucial. Aggressive infusion resuscitation, antibiotics, surgical removal of an infected area or necrotic tissue, drainage of purulent secretions and maintenance therapy are used as treatment [3-5].

The role of optimized antimicrobial therapy in pathogen clearance and mortality reduction is not currently in doubt. Parenteral administration of antibiotics is administered as soon as possible after taking blood samples, biological fluids and damaged tissues for gram staining and sowing. Early empirical therapy initiated immediately after suspicion of sepsis is important and can be crucial. The choice of antibiotic requires a reasonable assumption based on: the anticipated source of infection (e.g. pneumonia, urinary tract infection); clinical conditions; the proven or suspected presence of pathogens and their sensitivity characteristics characteristic of the hospital department or facility; and previous results of bacteriological sowing.

Typically, a wide range of gram-positive and gram-negative antibacterial are used first; patients with compromised immunity should also receive an empirical antifungal agent. There are many possible starting points for treatment; if available, knowledge 
about the specifics of the pathogens in the institutional environment and their susceptibility to antibiotics (antibiotics) should be used to select an empirical treatment. In general, typical antibiotics for empirical effects on Gram-positive bacteria are vancomycin and linezolid. Empirical therapy against gram-negative bacteria is more feasible and includes broad-spectrum penicillin's (e.g. piperacillin/tazobactam), 3rd or 4th generation cephalosporins, imipenem and aminoglycosides. The initial broad spectrum of action is reduced depending on the pathogen and its sensitivity to antibiotics [6].

The following principles of antibiotic therapy for sepsis are currently recommended (International Guidelines for Management of Sepsis and Septic Shock: 2016) [7,8]:

- Intravenous administration of antimicrobial agents should be initiated immediately after the identification of the pathogen and/or within 1 hour of the onset of the first symptoms of sepsis/septic shock (strong recommendation, moderate quality of evidence, evaluation applicable to both conditions).

- Empirical antimicrobial therapy is recommended in patients with sepsis/septic shock, including at least two classes of broad-spectrum antibiotics to treat a wider range of microorganisms or suspected pathogen, including bacteria, potential fungi and viruses (strong recommendation, moderate quality of evidence).

- Correction, in the form of a narrowing of empirical antibiotic therapy, should be made in the case of identification of the pathogen and its sensitivity and/or in the case of clinical improvement (best practice recommendation - British Pharmacological Society (BPS).

- $\quad$ Prophylactic prescribing of antibacterial in patients with severe inflammatory diseases of non-infectious genesis (severe pancreatitis, thermal burns of the skin, etc.) is not recommended (best practice recommendation - BPS).

- The dosing strategy for antimicrobial agents should be based on generally accepted pharmacokinetic/pharmacodynamic principles and should take into account the function of the organs and some specific features of antibacterial agents in patients with sepsis or septic shock (best practice recommendation - BPS).
- In the case of septic shock, initial empirical combined antibiotic therapy (using at least two antibiotics of different classes) should be directed towards a more likely range of pathogens (poor recommendation, poor quality of evidence).

- $\quad$ Routine clinical practice does not recommend combined antibiotic therapy for neutropenic fever/bacteriemia (strong recommendation, moderate quality of evidence).

- Combination antibacterial therapy is not recommended for the ongoing treatment of most other serious infections, including bacteriemia and sepsis without signs of shock (poor recommendation, poor quality of evidence).

- Combination antibacterial therapy is not recommended for routine treatment of neutropenic fever/bacteriemia (strong recommendation, average quality of evidence).

- In cases where combined antimicrobial therapy was originally used to treat septic shock, it is recommended that it be de-escalated or discontinued within the first few days in response to clinical improvement and/or proof of infection resolution. This applies to both etiotropic (positive culture of the pathogen) and empirical (in the case of negative bacteriological research) antibiotic therapy (best practice recommendation - BPS).

- Adequate duration of antibiotic therapy for the majority of infections associated with sepsis/septic shock is 7-10 days (poor recommendation, poor quality of evidence).

- $\quad$ Longer-term antibacterial use may be warranted in patients with slow clinical response to therapy; bacteriemia caused by Staphylococcus aureus; some fungal and viral infections; and patients with neutropenia (poor recommendation, poor quality of evidence).

- Shorter courses of antibiotic therapy may be available for certain patients, for example, in patients with rapid clinical response, and after adequate remediation of the source of infection in case of abdominal/ urinary sepsis or uncomplicated pyelonephritis (poor recommendation, poor quality of evidence).

- A daily assessment of the possibility of de-escalation of antimicrobial therapy in patients with sepsis/septic shock is recommended (best practice recommendation - BPS).

- $\quad$ Procalcitonin levels can be used to assess the duration of antimicrobial therapy in patients with sepsis (poor recommendation, poor quality of evidence) $[7,8]$. 
Perspective possibilities of sepsis curing, using drugs that activate the cholinergic anti-inflammatory pathway

Cholinergic stimulation, as we established in 1987 [9] and in subsequent studies, significantly reduces the mortality of albino mice from sepsis caused by intraperitoneal or intrapulmonary administration, respectively of E. coli and P. vulgaris [9-13]. Thus, the cholinergic anti-inflammatory mechanism has been discovered in 1987 [9], named «cholinergic anti-inflammatory pathway» in 2000 [14] after the research its implementation at the organismal, cellular and subcellular levels [10,13-15]. It should be noted that in 1995 it was proved the possibility of cholinomimetics for emergency activation of antimicrobial resistance of the organism in sepsis $[10,11]$. In the future, the study of the cholinergic antiinflammatory pathway caused by the action of acetylcholine on $\alpha 7 n$-acetylcholine receptors ( $\alpha 7 n A C h R s$ ) cells of the monocytemacrophage system (MMC), followed by inhibition of the production by the cells of pro-inflammatory cytokines (TNF- $\alpha$, IL-1 $\beta$, IL6 ) and reduced mortality from sepsis were devoted hundreds of articles various authors [12-22]. Reduced production of TNF- $\alpha$, IL-1 $\beta$, IL-6 (anti-inflammatory effect occurrence) for cholinergic anti-inflammatory pathway is provided kinase JAK2, transcription factor STAT3, NF-kB transcription factor) [14,19-23].

We have established on outbred mice that that acetylcholine chloride in a dose of $20 \mathrm{mg} / \mathrm{kg} 6 \mathrm{~h}$ after subcutaneous injection significantly reduces mortality of mice from sepsis induced by intraperitoneal injection of $2 \times 10^{9} \mathrm{CFUs}$ diurnal culture of E. coli in $2.0 \mathrm{ml}$ of saline and the blood levels of proinflammatory cytokines TNF- $\alpha$, IL-1 $\beta$, and IL-6 [18].

It was established in experiments on noninbred mice that activation of $\alpha 7 \mathrm{n}$-acetylcholine receptors ( $\alpha 7 \mathrm{nAChR})$ by anabasine in single doses of 1.0 and $5.0 \mathrm{mg} / \mathrm{kg}$ for $2 \mathrm{~h}$ before modeling sepsis (intraperitoneal injection of $2 \times 10^{9}$ CFUs diurnal culture of E. coli) cause a significant dose-dependent reduction of mortality of mice due to a decrease in the amount of proinflammatory cytokines TNF- $\alpha$, IL-1 $\beta$ and IL- 6 in the blood. Anabasine in single doses of 0.1 $\mathrm{mg} / \mathrm{kg}$ had no significant impact on the studied parameters [24].

The same action is taken by reversible inhibition of cholinesterase and nicotine on mouse mortality and blood levels of proinflammatory cytokines during the early phase of sepsis. So, the experiments on outbred albino mice have shown that Proserpine (reversible cholinesterase inhibitor) and nicotine (nicotinic re- ceptor agonist) in a equivalent dose of $0.2 \mathrm{DL}_{50}$ injected $2 \mathrm{~h}$ before sepsis induction significantly reduced animal mortality from experimental infection due to reduction of blood concentrations of proinflammatory cytokines TNF- $\alpha$, IL-1 $\beta$ and IL-6 [25].

The stimulation of nicotinic and muscarinic cholinoreceptors (nAChR, mAChR) in outbred albino mice with nicotine and aceclidine, respectively, in single equilethal doses $0.5 \mathrm{DL}_{50} 6 \mathrm{~h}$ before sepsis induction (intraperitoneal injection of $2,5 \times 10^{9} \mathrm{CFUs}$ diurnal culture of $E$. coli) significantly reduced animal mortality due to a decrease in blood concentrations of proinflammatory cytokines IL$1 \beta$, IL-6, and MIP-2. Stimulation of mAChR (injection of aceclidine) stimulated the neutrophilic phagocytic and metabolic activity. Realization of the cholinergic anti-inflammatory pathway (stimulation of the peripheral nicotinic cholinoreceptors, in particular, $\alpha 7 \mathrm{nAChR}$ and central muscarinic holoreceptors (in particular, m1AChR) was modulated by stimulation of the mAChR of the phagocytic monocytic system cells [26].

The activation of $\alpha 7 \mathrm{nAChR}$ with anabasine $\left(0.5 \mathrm{LD}_{50}\right)$ and the use of antibodies to TNF- $\alpha(1 \mathrm{mg} / \mathrm{kg}) 2 \mathrm{~h}$ before sepsis modeling significantly reduces mortality of mice from experimental sepsis due to a decrease in the blood concentration of TNF- $\alpha$, IL-1 $\beta$, and IL-6. After combined administration of anti-TNF- $\alpha$ antibodies and anabases, an additive effect was observed [22]. The same influence caused the effect of m1AChR agonist (TBPB) and $\alpha 7 \mathrm{nAChRs}$ agonist (GTS-21). Combined treatment with TBPB and GTS-21 determined their additive effect [23].

When the cholinergic anti-inflammatory pathway is realized, in addition to the excitation of $\alpha 7 n A C h R s[12,15,27,28]$, which cause the effects already mentioned, nAChRs activation of the brain substance of the adrenal glands and sympathetic ganglia occurs, which leads to the production of epinephrine and norepinephrine (NE), which activation of MMS adrenergic receptors and reduce the production of pro-inflammatory cytokines [28]. At this N. vagus, releasing acetylcholine ( $\mathrm{ACh}$ ) in the celiac ganglion, causes excitation of the spleen nerve, the action of NE through its efferent fibers on T lymphocytes, the production of ACh by these lymphocytes, activation of ACh of $\alpha 7 \mathrm{nAChRs}$ of MMS cells of the spleen [15,28]. Epinephrine and NE probably activating the adrenergic receptors of cells of the MMS (direct action) [28], $\beta 2$-adrenergic receptors ( $\beta 2 \mathrm{ARs}$ ) of spleen T lymphocytes (indirect effect) [16], cause the same effect as activation of $\alpha 7 \mathrm{nAChRs}$, leading to reduction in 
the synthesis of proinflammatory cytokines by cells of the MMS $[15,17,21]$.

Experiments on random-bred albino mice showed that of $\beta 2$ adrenoreceptor agonist hexaprenaline sulfate significantly reduced mortality of mice from experimental sepsis (intraperitoneal administration of E. coli) in 4 and $24 \mathrm{~h}$ after modeling by reducing blood levels of proinflammatory cytokines TNF $\alpha$, IL-1 $\beta$, and IL-6. The antagonist of $\beta 2 \mathrm{AR}$ ICI-118,551 eliminated this effect [21]. The combined administration of NF- $\mathrm{KB}$ inhibitor (BAY 11-7082) and $\beta 2$-adrenoreceptors agonist have an additive effect [23].

The influence study of various drags on mortality of mice and the concentration of proinflammatory cytokines in blood at sepsis caused by $E$. coli showed that administration m-cholinomimetic (aceclydine), n-cholinomimetic (nicotine), reversible inhibitor of acetylcholinesterase (neostigmine methyl sulfate), n-cholinomimetic $\alpha 7 n A C h R s$ agonist (GTS-21), epinephrine hydrochloride, andromimetic $\beta 2$ ARs agonist (hexaprenaline sulfate) causes decrease in mice mortality in sepsis caused by the administration (i.p.) of $E$. coli $0157: \mathrm{H} 7$ and the concentration of TNF- $\alpha$, IL-1 $\beta$ and IL-6 in the blood in comparison with parameters at sepsis without use of drugs [29].

Thus, n-cholinomimetic ( $\alpha 7 \mathrm{nAChRs}$ agonists, nicotine), reversible inhibitor of acetylcholinesterase, andromimetic ( $\beta 2$ ARs agonists) and m-cholinomimetic (m1-cholinomimetics), along with a range of therapeutic measures, in particular, with prescription of antibiotics, can be considered as promising agents for the treatment of sepsis and septic shock.

\section{Bibliography}

1. Pruett SB., et al. "Innate immunity and inflammation in sepsis: mechanisms of suppressed host resistance in mice treated with ethanol in a binge-drinking model". Toxicological Sciences 117.2 (2010): 314-324.

2. Martin GS. "Sepsis, severe sepsis and septic shock: changes in incidence, pathogens and outcomes". Expert Review of Antiinfective Therapy 10.6 (2012): 701-706.

3. Singer M., et al. "The third international consensus definitions for sepsis and septic shock (sepsis-3)". Journal of the American Medical Association 315 (2016)::801-810.
4. Gyawali B., et al. "Sepsis: The evolution in definition, pathophysiology, and management". SAGE Open Medicine 7 (2019): 1-13.

5. Hotchkiss RS., et al. "Sepsis and septic shock". Nature Reviews Disease Primers 2 (2016): 16045.

6. Liang SY and Kumar A. "Empiric antimicrobial therapy in severe sepsis and septic shock: optimizing pathogen clearance". Current Infectious Disease Reports 17.7 (2015): 493.

7. Rhodes A., et al. "Surviving Sepsis Campaign: International Guidelines for Management of Sepsis and Septic Shock: 2016". Intensive Care Medicine 43.3 (2017): 304-377.

8. De Backer D and Dorman T. "Surviving Sepsis Guidelines: A Continuous Move Toward Better Care of Patients with Sepsis". Journal of the American Medical Association 317.8 (2017): 807808.

9. Zabrodskii PF. "Effect of armin on nonspecific resistance factors of the body and on the primary humoral immune response". Farmakologiia I Toksikologiia 50.1 (1987): 57-60.

10. Zabrodskii PF. "Variation in antiinfectious nonspecific resistance of the organism caused by cholinergic stimulation". Bulletin of Experimental Biology and Medicine 120.2 (1995): 809811.

11. Zabrodskii PF. "Change in the non-specific anti-infection resistance of the body exposed to cholinergic stimulation". Bulletin of Experimental Biology and Medicine 120.8 (1995): 164-166.

12. PF Zabrodskii. "Immunotoxicology of organophosphorus compounds". Saratov (2016): 289.

13. Zabrodskii PF and Mandych VG. "Immunotoxicology of xenobiotics". Saratov Military Institute of Biological and Chemical Safety (2007): 420.

14. Borovikova LV., et al. "Vagus nerve stimulation attenuates the systemic inflammatory response to endotoxin". Nature 405.6785 (2000): 458-462.

15. Bernik TR., et al. "Pharmacological stimulation of the cholinergic antiinflammatory pathway". Journal of Experimental Medicine 195. 6 (2002):781-788.

16. Bonaz BL and Bernstein CN. "Brain-gut interactions in inflammatory bowel disease". Gastroenterology 144.1 (2013): 36-49. 
17. Fernandez R., et al. "Neural reflex regulation of systemic inflammation: potential new targets for sepsis therapy". Frontiers in Physiology 15.5 (2014): 489.

18. Reardon C. "Neuro-immune interactions in the cholinergic anti-inflammatory reflex". Immunology Letters 178 (2016): 92-96.

19. Zabrodskii PF. "Effect of acetylcholine on mortality of mice from sepsis and proinflammatory cytokine production". Bulletin of Experimental Biology and Medicine 150.3 (2011): 340342.

20. Zabrodskii PF., et al. "Effect of $\alpha 7 n$-Acetylcholine Receptor Activation and Antibodies to TNF- $\alpha$ on Mortality of Mice and Concentration of Proinflammatory Cytokines During Early Stage of Sepsis". Bulletin of Experimental Biology and Medicine 159.6 (2015): 740-742.

21. Zabrodskii PF., et al. "Role of $\beta 2$-adrenoreceptors in adrenergic anti-Inflammatory mechanism in sepsis". Bulletin of Experimental Biology and Medicine 162.12 (2017): 718-721.

22. Zabrodskii PF., et al. "Combined Effects of M1 Muscarinic Acetylcholine Receptor Agonist TBPB and $\alpha 7 n$-Acetylcholine Receptor Activator GTS-21 on Mouse Mortality and Blood Concentration of Proinflammatory Cytokines in Sepsis". Bulletin of Experimental Biology and Medicine 162.6 (2017): 750-753.

23. Zabrodskii PF., et al. "Combined Effect of NF-кB Inhibitor and $\beta 2$-Adrenoreceptor Agonist on Mouse Mortality and Blood Concentration of Proinflammatory Cytokines in Sepsis". Bulletin of Experimental Biology and Medicine 162.6 (2018): 445448.

24. Zabrodskiı̌ PF., et al. "The effect of anabasine on mortality and concentration of proinflammatory cytokines in blood of mice at early stage of sepsis". Eksperimental'naia i klinicheskaia farmakologiia 77.11 (2014): 20-22.

25. Zabrodskii PF., et al. "Effects of reversible inhibition of cholinesterase and nicotine on mouse mortality and blood levels of proinflammatory cytokines during the early phase of sepsis". Bulletin of Experimental Biology and Medicine 152.5 (2012):600-602.

26. Zabrodskii PF., et al. "Role of nicotinic and muscarinic cholinoreceptors in the realization of the cholinergic anti-inflammatory pathway during the early phase of sepsis". Bulletin of Experimental Biology and Medicine 153.5 (2012):700-703.
27. Link A., et al. "Phosphodiesterase 4 inhibition but not beta-adrenergic stimulation suppresses tumor necrosis factor-alpha release in peripheral blood mononuclear cells in septic shock". Critical Care 12.6 (2008). R159.

28. Scanzano A and Cosentino M. "Adrenergic regulation of innate immunity: a review". Frontiers in Pharmacology 23.6 (2015): 171.

29. PF Zabrodskii. "The Influence of Various Drugs on Mortality of Mice and the Concentration of Proinflammatory Cytokines in Blood at Sepsis Caused by E. coli". Acta Scientific Microbiology 1.5 (2018): 11-14.

\section{Volume 2 Issue 11 November 2019 (C) All rights are reserved by Pavel Franzevich} Zabrodskii. 